\title{
Recent Developments in the Theory of Electron Cyclotron Waves
}

\author{
Emanuele Poli \\ Max-Planck-Institut für Plasmaphysik, \\ EURATOM Association, Garching bei München, Germany
}

Total Number of Pages: 28

Total Number of Figures: 4

Proofs to be sent to:

Dr. E. Poli

Max-Planck-Institut für Plasmaphysik

Boltzmannstr. 2, Geb. L5

85748 Garching bei München

Germany

Tel. No. +49 8932991671

Fax No. +49 8932992580

Electronic mail: Emanuele.Poli@ipp.mpg.de 


\title{
Recent Developments in the Theory of Electron Cyclotron Waves
}

\author{
Emanuele Poli \\ Max-Planck-Institut für Plasmaphysik, \\ EURATOM Association, Garching bei München, Germany
}

\begin{abstract}
Quantitative predictions of propagation, emission and absorption of electron cyclotron waves rely on a solid theoretical background and are routinely employed in the analysis and preparation of present and future fusion experiments. Nonetheless, open problems still exist and improvements are possible also within well-established models, to make them faster, more accurate or more general. This paper presents some of the recent advances in the theoretical investigation of electron cyclotron waves. Particular emphasis is put on the "standard" approach based on the short-wavelength approximation and linear or quasilinear computation of wave absorption, on the theory of electron Bernstein waves and on the applications of cyclotron heating and current drive for ITER.
\end{abstract}




\section{INTRODUCTION}

It is a common statement in the plasma-physics community that the theory of propagation, emission and absorption of electromagnetic waves in the electron-cyclotron (EC) frequency range is well established and that the uncertainty connected to numerical predictions is at a tolerable level. EC computer codes are routinely used to analyse experimental results and to plan new campaigns.

Indeed, our present knowledge looks pretty robust. The theory of EC waves and their applications to experiments are reviewed in classical papers [1,2]. Calculations based on the Wentzel-Kramers-Brillouin (WKB) model (i.e. on the short-wavelength approximation) for wave propagation and on linear theory for the description of power absorption and current generation are very fast and can be performed for arbitrary geometries, including equilibria and plasma profiles reconstructed from experimental data. A refinement in the description of the wave-particle interaction, employing a (more computationally demanding) quasilinear approach, is also successfully employed. Several features predicted by the theory of waveplasma interactions can be checked experimentally, sometimes with an astonishing precision, as it has been shown in recent reviews $[3,4]$. Possible disagreements can be often explained in terms of effects which do not directly involve wave physics, like the radial transport distributing the electrons across the region of interaction with the wave beam [5-8].

This does not mean, of course, that every problem has been solved. An intense research activity is being carried out in order to find solutions to open or controversial points, but also to extend the applicability of present models or refine them in order to make them faster or more accurate.

The aim of this paper is to present some of the recent advances in the theory, as they emerge from journal articles and from the contributions presented at the $14^{\text {th }}$ Joint Workshop on Electron Cyclotron Emission (ECE) and Electron Cyclotron Resonance Heating (ECRH) (Santorini Island, Greece, May 2006). Unavoidably, the spectrum of the topics discussed here is far from being complete. As a possible classification of the theory work done in the last few years, one can identify three big research areas, namely,

1. the basic theory of propagation, absorption and emission of EC waves;

2. the description of electron Bernstein waves; 
3. the applications of EC waves in view of ITER

(where the last two topics have of course a significant overlap with the first one). This rough scheme will be used as a guideline in this paper. At each step, some open questions in EC theory will be discussed. A survey of our current ignorance in the field shows [9] that several issues still need to be investigated or clarified. In this paper it will be attempted to highlight cases where some progress has been achieved, as well as to outline new problems which have recently emerged. Sec. II thus deals with the new developments in the description of propagation, absorption and current drive $(\mathrm{CD})$, in particular within the short-wavelength approximation and the linear and quasilinear theory. Electron Bernstein waves are treated in Sec. III, whilst the applications to ITER are discussed in Sec. IV. Finally, other topics are briefly summarised in Sec. V and the conclusions are drawn in Sec. VI.

Since this paper is conceived as a summary of some recent progress and not as a systematic review of EC theory, the mathematical formalism is sketched only insofar as necessary to understand the content of the new developments presented. The interested reader is referred to the original papers quoted in the References for more details.

\section{PROPAGATION, ABSORPTION AND CURRENT DRIVE}

As stated in the Introduction, numerical codes based on the short-wavelength approximation are worldwide employed as the fundamental tool for quantitative predictions of propagation, emission and absorption of EC waves. They can differ in the model chosen to describe the transverse beam structure (standard ray tracing [10-14], quasi-optical ray propagation [15-17], beam tracing [18-21]), the interaction with the plasma (weakly or fully relativistic treatment, linear or quasilinear absorption [22]) and the driven current (directly from the distribution function, from an adjoint model [23-25]) and several other approximations are done in the physics or in the numerical implementation. As we will see below, within this framework the understanding of the approximations used in different models is so detailed that discrepancies of the order of $10 \%$ can be investigated. Some issues involving a more refined physics description, which goes beyond the approach outlined above, are treated in Sec. II D. 


\section{A. Propagation}

In the WKB approximation, a simplification of the wave equation

$$
\nabla \times(\nabla \times \mathbf{E})-\frac{\omega^{2}}{c^{2}} \varepsilon \cdot \mathbf{E}=0
$$

(where $\varepsilon$ is the dielectric tensor, $\omega / 2 \pi$ is the wave frequency and $c$ is the speed of light) is achieved by expanding the wave field $\mathbf{E}$ in powers of the small parameter $\lambda / L$, where $\lambda$ is the wavelength and $L$ is the inhomogeneity scale of the medium. To the lowest order, this results in a set of Hamiltonian ray equations [10], each ray being described by its position $\mathbf{r}$ and wavevector $\mathbf{k}$ :

$$
\frac{\mathrm{d} \mathbf{r}}{\mathrm{d} \tau}=\frac{\partial H}{\partial \mathbf{k}} \quad \frac{\mathrm{d} \mathbf{k}}{\mathrm{d} \tau}=-\frac{\partial H}{\partial \mathbf{r}}
$$

In these equations, the Hamiltonian function is given by the dispersion relation obtained as the solvability condition for the wave equation in Fourier space. To the next order, under the assumption of moderate absorption, one obtains a transport equation for the wave amplitude. Higher-order equations of the WKB hierarchy are not employed, as they become more and more cumbersome. Since every ray is traced independently of the others, wave effects are not retained at this level. However, diffraction can be important for focused or highly collimated beams employed in many experiments. Extensions of standard ray tracing, which include these effects and still allow fast calculations, are usually achieved within the complex-eikonal approach [26, 27] (alternatively, one can assign the initial conditions for the rays such as to match asymptotically the diffractive solution in vacuum). In the complex-eikonal scheme, an imaginary part is added to the wave phase (eikonal) in order to describe the transverse profile of the beam. The beam width $W$ is assumed to be larger than the wavelength and smaller than the inhomogeneity scales of the plasma,

$$
\lambda \ll W \ll L
$$

Starting from this ansatz, two approaches have been developed. In the quasi-optical (QO) ray tracing [15], the ray equations are linked together to take into account diffraction effects. This is achieved by expanding the (complex) dispersion relation in powers of $\lambda / W \sim \mathbf{k}^{\prime \prime} / \mathbf{k}^{\prime} \ll$ 1 , where $\mathbf{k}^{\prime}=\operatorname{Re}(\mathbf{k})=\operatorname{Re}(\nabla S)$ and $\mathbf{k}^{\prime \prime}=\operatorname{Im}(\mathbf{k})=\operatorname{Im}(\nabla S), S$ being the wave phase. The dispersion relation $H$ used in the ray equations (1) can be explicitly written as

$$
H\left(\mathbf{k}^{\prime}+i \mathbf{k}^{\prime \prime}\right) \simeq H\left(\mathbf{k}^{\prime}\right)+i \mathbf{k}^{\prime \prime} \cdot \frac{\partial H}{\partial \mathbf{k}^{\prime}}-\frac{1}{2} \mathbf{k}^{\prime \prime} \mathbf{k}^{\prime \prime}: \frac{\partial^{2} H}{\partial \mathbf{k}^{\prime} \partial \mathbf{k}^{\prime}}=0 .
$$


The real part (first and third term) of the left-hand side of Eq. (3) is used as Hamiltonian function in the QO ray equations (the first term is the standard Hamiltonian of geometrical optics). The imaginary part of Eq.(3) allows the closure of the system introducing a coupling between adjacent rays. This method has been chosen for implementation in the last-born in the family of EC codes, namely the code GRAY [28]. A new algorithm has been devised to advance the coupled ray equations. It allows fast calculation of the beam trajectory with a good numerical accuracy. Other features of GRAY are highlighted in Sec. II B. Generally speaking, the QO approach has the advantage of preserving the description of the beam as a bundle of rays. The power deposition profile is a simple superposition of the power absorbed from each ray, each satisfying separately the resonance condition. An alternative method is given by the (paraxial) beam tracing $[18,19]$, where the inequality $(2)$ is explicitly used to reduce the wave equation to a set of ordinary differential equations for a reference ray and a set of parameters connected to the width of the beam and the curvature of the phase front. Since a single ray is calculated, the equations can be advance by standard (e.g. RungeKutta) algorithms; moreover, the number of equations to be solved is intrinsically lower than in the QO method. An obvious disadvantage is that the calculation of the absorption profile requires a method for projecting the beam onto the EC resonance, since the amplitude transport equation is solved on the reference ray only. The approximation introduced by neglecting the $k_{\|}$-spectrum in the caclulation of absorption $\left(k_{\|}\right.$is the component of $\mathbf{k}$ parallel to the magnetic field) has been investigated recently $[29,30]$ and has been shown to give accurate results for beam widths larger than $1 \mathrm{~cm}$.

Some considerations about inequality (2) must be added. This ordering is reasonable for many standard applications. It is not difficult, however, to imagine situations where it fails, as already pointed out [9]. A first example is given by warm-plasma effects leading to a strong variation of the dispersion properties of the plasma within the resonance layer [31], which has usually a width comparable to the beam width. A second example is given by the case of propagation oblique to the resonance layer, for instance in scenarios with large toroidal injection angle or launch from positions far from the equatorial plane. When a part of the beam enters the resonance much earlier than the other part, the descriptions based on an eikonal approach fail. Similar considerations apply to the case of propagation close to cutoffs, as those typical for mode-conversion schemes, where the dispersive properties of the plasma undergo again fast variations across the beam front. A full-wave approach, at least 
close to the resonace layers, could become necessary in those cases. How to incorporate this kind of effects within a WKB approach is still an open issue. The research activity aiming at an accurate description of beam propagation without resorting to full-wave techniques is still ongoing $[32,33]$.

\section{B. Absorption and Current Drive}

The formal expression for the hot-plasma dielectric tensor has been derived long time ago [34]. As is well known, the linear response of the plasma to the electromagnetic field of the wave can be calculated immediately in Fourier space from the Vlasov equation. Writing the distribution function as $f=n_{0} f_{0}+f_{1}$ (where $n_{0}$ is the equilibrium density, $f_{0}$ is the unperturbed distribution function and $f_{1}$ is the perturbation), one has

$$
f_{1}(\mathbf{p}, \omega, \mathbf{k})=e n_{0} \int_{0}^{\infty} \mathrm{d} \tau e^{i \Phi(\tau)}\left[\left(\frac{\partial f_{0}}{\partial p_{\perp}}+\frac{N_{\|}}{\gamma m c} F\right) \frac{\mathbf{p}^{\prime}}{p_{\perp}}+\frac{F}{p_{\perp}}\left(1-\frac{\mathbf{p}^{\prime} \cdot \mathbf{N}}{\gamma m c}\right) \hat{\mathbf{z}}\right] \cdot \mathbf{E},
$$

where $N_{\|}=c k_{\|} / \omega$, the static magnetic field $\mathbf{B}$ is in the $z$-direction, $\gamma=\sqrt{1+p^{2} / m^{2} c^{2}}$ is the relativistic Lorentz factor ( $m$ is the electron mass) and

$$
\begin{aligned}
& \Phi(\tau)=\left(\omega-k_{\|} v_{\|}\right) \tau+\frac{k_{\perp} p_{\perp}}{m \omega_{c}}\left[\sin \left(\phi-\omega_{c} \tau / \gamma\right)-\sin \phi\right], \\
& \mathbf{p}^{\prime}=p_{\|} \hat{\mathbf{z}}+p_{\perp}\left[\cos \left(\phi+\omega_{c} \tau / \gamma\right) \hat{\mathbf{x}}-\sin \left(\phi+\omega_{c} \tau / \gamma\right) \hat{\mathbf{y}}\right], \\
& F=p_{\perp} \frac{\partial f_{0}}{\partial p_{\|}}-p_{\|} \frac{\partial f_{0}}{\partial p_{\perp}},
\end{aligned}
$$

$\phi$ being the gyroangle and $\omega_{c}=e B / m$ the cyclotron frequency. The dielectric tensor is defined as $\boldsymbol{\varepsilon}=\mathbf{I}+4 \pi i \boldsymbol{\sigma} / \omega$, where $\mathbf{I}$ is the unitary tensor and the conductivity tensor $\boldsymbol{\sigma}$ is defined through the relation $\mathbf{j}=\boldsymbol{\sigma} \cdot \mathbf{E}$, with

$$
\mathbf{j}=-e \int \mathrm{d}^{3} \mathbf{p} f_{1} \frac{\mathbf{p}}{\gamma m}
$$

where $f_{1}$ is given by Eq. (4).

Even under the assumption of a relativistic Maxwellian distribution function $f_{0}$, it is not trivial to find the best method for dealing with the integrals in Eqs. $(4,5)$. To simplify the time integration in Eq. (4), the exponential factor $\exp [i \Phi(\tau)]$ can be written using Jacobi's identity for the Bessel function $J_{n}(z)$,

$$
e^{i z \sin \phi}=\sum_{n=-\infty}^{\infty} J_{n}(z) e^{i n \phi}
$$


which introduces an infinite sum over the cyclotron harmonics. Using this identity, the oscillating factor can be written for each $n$ as $\exp \left[i\left(\omega-k_{\|} v_{\|}-n \omega_{c} / \gamma\right) \tau\right]$. The integration over $\tau$ gives then immediately the cyclotron-resonance condition

$$
\gamma-N_{\|} p_{\|} / m c-n \omega_{c} / \omega=0
$$

The research on the methods to be used in order to reduce the computational effort needed to solve these equations is still ongoing. The goal is, on one hand, to find faster algorithms to be employed in the solution of conventional problems, like the calculation of linear emission and absorption coefficients, and on the other hand to extend the models beyond their original range of applicability, as is the case for the propagation of Bernstein waves, where the parallel wavenumber $N_{\|}$can be larger than unity (this second topic is addressed in Sec. III).

A new approach to the calculation of the relativistic dispersion relation for EC waves has been proposed by D. Farina [35]. Generalising a similar approach adopted in the weakly relativistic case [36], the Bessel functions arising from Eq.(6) are expanded in power series of their argument $k_{\perp} v_{\perp} /\left(\omega_{c} / \gamma\right)$ and the index $n$, denoting the harmonic number, is combined with the index $k$, denoting the order of this Larmor-radius expansion of the Bessel functions, into a single index $l=n+k$, so that the relativistic dielectric tensor can be presented as a series over $l$, in which each element is a sum on the harmonic number number $n=$ $0, \mp 1, \ldots, \mp l$. For the fully relativistic case, the integration of the real part of the integral over the parallel component of the momentum must be performed numerically, whilst the integration over the imaginary part can be simplified employing recurrence rules, which allow one to compute higher-order terms in the Larmor-radius expansion as a linear combination of lower-order terms. In the weakly relativistic approximation, the inequality $p / m c \ll 1$ can be employed to expand $\gamma$ and the relativistic Maxwellian distribution to the leading order (see the paper [35] for details), so that the integrals can be expressed in terms of the standard Shkarofsky functions [36, 37]. This approach is particularly suited for numerical implementation and is used in GRAY to calculate the absorption coefficient for both the fully relativistic and weakly relativistic cases.

In general, the problem of which approximations are meaningful for a proper calculation of the dielectric tensor is obviously related to the problem at hand. For reactor-grade plasmas, for instance, a calculation of the tensor based on a weakly relativistic approximation 
may be inadequate (see also Sec. III). In the weakly relativistic approximation, resonant interaction is possible for $\omega_{c} / \omega \geq 1-N_{\|}^{2} / 2$, which requires slightly higher magnetic field than in the fully relativistic case $\omega_{c} / \omega \geq \sqrt{1-N_{\|}^{2}}$. The impact for ITER, for instance, has been evaluated within a benchmarking activity of different EC codes [38]. First of all, the position of the peak of the absorption profile shifts to the high-field side in the weakly relativistic approximation. Moreover, the weakly relativistic approach underestimates the range of resonant parallel momentum of the electrons interacting with the wave (see Fig. 1), which can result in a decrease of the current drive efficiency. These effects are non-negligible if calculations aim at an accuracy of the order of $10 \%$, as mentioned before. Another approximation in the calculation of the current-drive efficiency which potentially leads to errors of the same order of magnitude is the simplification of the polarisation term in the quasilinear diffusion operator to include only one polarisation of the wave field, as done e.g. in Cohen's model [24]. More recent models [25, 28] include the full polarisation term. It has been shown that a pile-up of small errors due to different approximations can lead to significant differences in the resulting predictions [39]. A final point should be stressed here. A further approximation done in all codes using a linear model for absorption and current drive is the high-velocity limit employed to simplify the collision operator, which is justified only if the velocity of the resonant particles is much larger than the thermal velocity. For high-temperature plasmas, as those envisaged for the ITER core, this approximation is questionable. In this respect, linear simulations performed for ITER scenarios with offaxis deposition for NTM stabilisation (see Sec. IV) have shown a good agreement [38] with quasilinear simulations performed with the CQL3D code [40]. Under different conditions, for example higher electron temperature and smaller magnetic well, the differences can be much larger [41], also because of the momentum-conserving collision operator implemented in CQL3D (conservation of parallel momentum is usually not implemented in linear calculations; however, its inclusion is possible also for this case [42]). Generally speaking, high-power EC experiments leading to substantial nonthermal distributions must be interpreted by means of Fokker-Planck calculations, but the role of quasilinear effects in large devices is usually insignificant (for a discussion of this point, which goes beyond the scope of this paper, see e.g. Ref. [4]). 


\section{Emission}

As the emission of EC waves (ECE) is routinely used to diagnose the temperature of the plasma electrons, several codes used for the calculation of absorption and current drive contain routines that solve the radiative-transfer equation [43] along the rays.

Two examples of the level of sophistication reached by ray tracing codes for this goal are given by the BRT (now renamed TRAVIS) code [44] and by the GENRAY code [45]. Important features common to both codes are the flexibility in the specification of the electron distribution function as well as the possibility of going beyond the cold-plasma approximation in the determination of the ray trajectory. In particular, a very detailed analysis of the localisation and the theoretical limit on the resolution of the measurements is included in BRT/TRAVIS, for both low-field-side and high-field-side observation [46].

As EC power losses can give a significant contribution to the energy balance of a reactor plasma [47], fast and reliable procedures that can be implemented in transport code are needed. To this aim, Fokker-Planck calculations are too time consuming. A fully-relativistic "quasi-exact" analytic calculation of the absorption coefficient, which uses Bateman's expansion for the product of two Bessel functions, has been used [48] to assess the accuracy of asymptotic expressions that have been given earlier $[49,50]$. This analysis shows that these approximations can be usefully applied for calculating EC losses.

However, since the EC emission can be strongly influenced by non-thermal electron population, a detailed knowledge of the electron distribution function is sometimes crucial. For the case of ECCD-generated suprathermal electrons under ITER conditions, it has been shown through a coupling of the beam-tracing code TORBEAM [51] and the Fokker-Planck code RELAX [52] that suprathermal electrons can lead to a radiation profile up to $20 \%$ higher in the plasma core $[53,54]$ (this is due to the fact that, very close to the axis, the interaction volume for the EC power can be pretty small and the Harvey criterion for the onset of quasilinear effects [55] can be approached even in a large device like ITER). The impact of EC radiation on ECCD has to be assessed. Anyway, this enhanced ECE radiation should play no significant role in the total power balance. 


\section{Beyond the standard approximations}

Nonlinear effects connected with the propagation and absorption of electromagnetic waves in plasmas are not usually taken into account in the analysis of experimental results, since the power densities which can be obtained from presently available gyrotrons are believed to be too small. It has recently been suggested, however, that nonlinear effects could have an impact on absorption and current drive in present-day tokamaks $[56,57]$. The parameter determining the influence of nonlinear effects on the dynamics of the electrons interacting with the beam [58] is the ratio between the electron time of flight across the beam, $\tau_{f}$, and the period of nonlinear oscillations inside the beam, $\tau_{b}$, which scales as $\epsilon_{N L}=\tau_{f} / \tau_{b} \sim$ $P^{1 / 4} B^{1 / 2} W^{1 / 2} v_{\perp} / v_{\|}$, where $P$ is the beam power. A nonlinear broadening of the absorption profile for perpendicular injection could be screened by the radial transport of the charge carriers mentioned above. An effect on the current drive could be seen if the beam width in the toroidal direction could be made much broader than in the poloidal direction, for instance by aiming the beams at neighbouring toroidal positions. An experimental verification of these predictions is still missing.

A different situation where nonlinear interaction of EC waves and plasmas could be of practical importance at realistic power levels is the so-called induced transparency which can be realised by introducing a nonlinear change in the dielectric properties of the plasma by means of a three-wave coupling. Electromagnetically induced transparency could be used to access linearly opaque regions of the plasma [59,60]. More recently, it has been proposed to use parametrically induced transparency to detect radiation of Bernstein waves from the core of the plasma by inducing a tunneling through the evanescent region [61]. This scheme could hence be an alternative to the $B X O$ detection (cf. Sec. III). Since the nonlinear coupling is small, the signal is predicted to be quite low. However, it could be detectable since it is detuned from the first and the second EC harmonics and the noise level would be small. Experiments to verify this hypotesis are planned.

A result which seem to contradict the well-established picture given in the previous sections has been obtained in the last months by means of the full-wave code STELEC [62], which does not employ the WKB approximation nor one of its refinements discussed in Sec. II A. The upper-hybrid resonance is seen to have a significant impact, not predicted by the other codes, on the propagation of EC waves, in particular when the wave is injected at 
the first harmonic of the ordinary mode. Anomalous mode conversion could lead to reduced absorption and shine-through of the beam. In a recently updated STELEC code version [63], the full set of plasma response currents has been included and EC beam shine through was strongly reduced, while again strongly supporting the crucial role of $O$-mode and $X$ mode coupling at $O$-mode injection from the low-field side. Additional full-wave (analytic or numeric) investigation of this issue will help clarify this point.

The importance of an analytic approach to some of the problems where the WKB approximation is expected to fail has been demonstrated in an instructive way in the analysis of the effects of two-dimensional inhomogeneity on mode conversion in toroidal plasmas [6466]. This method, which could possibly give new insights also in some problem mentioned in Sec. II A, is discussed in Sec. III.

\section{ELECTRON BERNSTEIN WAVES AND MODE CONVERSION}

Electron Bernstein waves (EBW) offer a way to access the core of overdense plasmas, which would be otherwise opaque to standard low-harmonic $O$-mode and $X$-mode waves. These high densities are typical, in particular, for stellarators and spherical tori. For this reason EBW, which have been known for several decades [67, 68], are at present subject of intense investigation. EBW are electrostatic waves sustained by the cyclotron motion of the electrons, so that their wavelength is of the same order as the electron Larmor radius. Being inherently a (hot) plasma effect, these waves do not exist in vacuum and cannot be excited directly from an antenna. However, they can couple to extraordinary waves, which possess a component of the wave field along the wave vector. This is particularly true close to a resonance, where the phase velocity of the wave becomes smaller and smaller and can finally match the electron thermal speed. Indeed, the most successful scheme for generating EBW is through oblique injection of a wave beam in the ordinary mode, in such a way that the turning point is as close as possible to the cutoff layer. There, the wave is converted into an $X$-mode wave which couples to a Bernstein wave at the upper-hybrid resonance $(O X B$ scheme [69]). EBW can hence be used as a diagnostic tool (e.g. by detection through the inverse $B X O$ scheme [70]) as well as for heating and current-drive purposes. It has been shown [71-74] that a parallel current can be generated through both the Ohkawa mechanism [75], which is particularly suited for the outer part of spherical tori, where the 
trapped-particle fraction is very high, as well as through the Fisch-Boozer scheme [76] in the passing domain.

The evaluation of the dielectric tensor for EBW requires some care. First of all, it is known that relativistic effects can be important even for the case of oblique propagation in regions where the cyclotron harmonics are far from the wave frequency (see e.g. Eq.(2.3.81) of Ref. [1] and discussion there). Moreover, the assumption $N_{\|}<1$, valid for "cold" EC waves, cannot be made for EBW. This is due to the fact that their phase velocity is of the order of the electron thermal velocity, i.e. $N_{\perp}$ and hence $N_{\|}$can be much larger than unity. It has been shown [77] that in the weakly relativistic limit it is possible to express the dielectric tensor in a compact form without making any assumption on the size of $N_{\|}$. One way to show this is to use the second of Trubnikov's formulae [1, 34], which can be obtained from Eqs. $(4,5)$ taking a relativistic Maxwellian distribution $f_{0}$ and performing the integral over the momenta. Employing asymptotic limits for the modified Bessel functions of second kind appearing in the resulting time-history integral, the exponential factor in Eq. (4) can be brought to a form which can be expanded using the equalities

$$
\exp \left[\frac{\lambda \cos y t}{1-i t}\right]=\sum_{p=0}^{\infty} \sum_{q=0}^{\infty}\left[\frac{\lambda}{2(1-i t)}\right]^{p+q} \frac{e^{i(p-q) y t}}{p ! q !}=\sum_{n=-\infty}^{\infty} \sum_{m=|n|}^{\infty} \frac{(\lambda / 2)^{m}}{\left(\frac{m+n}{2}\right) !\left(\frac{m-n}{2}\right) !} \frac{e^{i n y t}}{(1-i t)^{m}}
$$

where the double sum is restricted to even values of $m+n$. This form allows one to perform the integral over $\tau$ in terms of the generalised Shkarofsky functions [78], which can be recursively related to one another [79].

The validity of the weakly relativistic approximation can be obtained from a comparison with a fully relativistic treatment. This has been done recently by evaluating the fully relativistic dielectric tensor employing a method based on the relativistic generalisation [80] of the concept of plasma dispersion function [81], known from the non-relativistic case. The fully relativistic dispersion function has been evaluated for arbitrary values of temperature and wave number [80] and its analytic properties have been studied. A comparison with the weakly relativistic treatment shows a difference larger than $5 \%$ between the two dispersion relations for temperatures above approximately $20 \mathrm{keV}$ for the fundamental harmonic, above $10 \mathrm{keV}$ for the second harmonic and above $5 \mathrm{keV}$ for the third harmonic. Under experimental conditions, it has been observed that relativistic effects are important for EBW, since the ray trajectories can be different from those computed in the non-relativistic limit $[73,82]$. As a consequence, the position where the wave is absorbed changes, as well as the current 
drive efficiency, because of the different value of $N_{\|}$. In general, future work may entail detailed testing of approximate dispersion relations against the more time-consuming nonapproximate numerical solutions.

The generation efficiency of EBW in $O X B$ schemes depends basically on the efficiency of mode conversion at the cutoff layer. Although the importance of $2 \mathrm{D}$ effects for conversion of EC waves into EBW at the upper-hybrid resonance was pointed out long time ago [83], the $O X$ mode-conversion efficiency has usually been calculated on the basis of slab-geometry models. Close to the mode-conversion region, the dispersion relation can be written in the form [84] $N_{\perp}^{2}=2 \mathcal{P}\left(\mathcal{L}-N_{\|}^{2}\right) / \mathcal{L}$, where the cold-plasma parameters $\mathcal{P}$ and $\mathcal{L}$ are defined as [85] $\mathcal{P}=1-\omega_{p}^{2} / \omega^{2}$ and $\mathcal{L}=1-\omega_{p}^{2} / \omega\left(\omega+\omega_{c}\right)$. If the density gradient points in the same direction as the gradient of the magnetic field (one-dimensional geometry), the two surfaces $\mathcal{P}=0$ and $\mathcal{L}=N_{\|}^{2}$ are parallel and the conversion efficiency is determined by the width of the evanescent region between them. In a tokamak, however, this situation occurs only in the midplane and the two surfaces, in general, cross (see Fig. 2). In this case, the wave equation can be reduced, in the limit $\lambda / L \ll 1$, to a system of two first-order partial differential equations for the relevant components of the electric field [64-66] which can be solved analytically. The most pronounced features of these solutions are the existence of an "optimal" beam shape leading to total mode conversion and the asymmetry in the transformation efficiencies from $O$ to $X$ mode and backward. The impact of these $2 \mathrm{D}$ effects on the transmission coefficient is of course very pronounced if the angle $2 \alpha$ between the directions of $\nabla \mathcal{P}$ and $\nabla \mathcal{L}$ is large, but can be significant (about 20\%) also for values of $\alpha$ around $5^{\circ}$, cf. Fig. 4 of Ref. [64]. These solutions can also be exploited to prescribe the geometric-optics field past the singular layer [65]. It has also been shown that inclusion of the poloidal component of the static magnetic field does not change the structure of these equations if the poloidal component is much smaller than the toroidal one [66]. These results are extremely important for a proper comparison with ray-tracing calculations.

\section{NTM STABILISATION AND APPLICATIONS TO ITER/DEMO}

As for many areas of fusion research, some developments in the EC theory are induced by the need for reliable predictions of ITER scenarios. ECRH and ECCD are envisaged at ITER for core heating and H-mode access, for current profile control and for the control of 
magnetohydrodynamic instabilities, in particular neoclassical tearing modes (NTMs) [86]. The waves can be injected from an equatorial launcher (EL), located in the midplane of the tokamak, which delivers the EC power to the core plasma, and an upper launcher (UL), located in the top of the vessel, which should aim at the outer part of plasma, basically for NTM stabilisation. NTMs have been shown to cause significant degradation of the plasma confinement in present-day tokamaks. For ITER, a fully developed NTM could lead to a decrease of the fusion gain $Q=P_{\text {fusion }} / P_{\text {aux. heating }}$ of the order of $25 \%$ for a $(m=3, n=2)$ mode (where $m$ and $n$ denote the poloidal and toroidal mode numbers), and up to $50 \%$ for a $(2,1)$-mode [87], which is unacceptable in order to achieve the experimental goals of the machine. EC waves are particularly attractive for the purpose of NTM suppression since they can be deposited in a narrow region around the resonant surface, allowing replacement of the missing bootstrap current inside the island and also local changes of the plasmacurrent profile. For this reason, the development of the UL, in particular, has stimulated an intense activity [88], connected, on the one hand, with the necessity of evaluating the impact of EC waves on the dynamics of NTMs and establishing criteria for complete stabilisation (in passing, it should be noted that full stabilisation of NTMs is desirable only if the needed EC power does not lead itself to a too drastic decrease of the value of $Q$ ) and, on the other hand, with the more EC-specific task of calculating with high accuracy the location and the profile of the driven current.

Concerning the first point, the greatest difficulty is at present our incomplete undestanding of the physics governing the dynamics of the NTM, in particular at small island widths. In other words, we are not in a position to compute the island evolution from saturation till complete suppression. To be on the safe side, a lower bound for the amount of EC-driven current which will be necessary to completely stabilise the mode has been evaluated. On the basis of the modified Rutherford equation, the criterion for full NTM stabilisation has been formulated as $\eta_{E C C D} \equiv j_{E C C D} / j_{b s}>1.2$, where $j_{E C C D}$ is the driven peak current density and $j_{b s}$ is the missing bootstrap current density [89]. Partial stabilisation at reduced EC power could be preferable if NTMs are always present, in order not to deteriorate too much the fusion gain $Q$ [87], as mentioned above. Moreover, modulation capabilities should be retained if the width of the driven-current profile exceeds the island width [90, 91].

The accuracy that can be achieved in the theoretical prediction of the position and the peak value of the driven current density is important, since the efficiency of NTM stabilisa- 
tion by ECCD crucially depends on our ability of aligning the EC current with the location of the rational surface. The reliability of the calculations of ECCD profiles for typical ITER parameters has been tested through a benchmarking of several ray/beam tracing codes [38]. As discussed in Sec. II B, the fully relativistic absorption coefficient can slightly differ from weakly relativistic calculations, cf. Fig. 3. The total driven currents are within $5 \%$ in most of the codes, for both linear and quasilinear models (as mentioned above, this picture could change for different scenarios involving the plasma core; moreover, a detailed comparison of the current profiles will require some extension of the benchmarking exercise). For the sake of completeness, we quote here the codes involved in the benchmarking: TORAY-GA [92, 93], TORAY-FOM [92, 94], GENRAY [95], CQL3D [40], BANDIT-3D [96], TORBEAM [51], OGRAY [97] and GRAY [28].

These results give us a good confidence in the present predictions of ECCD profiles for the ITER UL. Indeed, to evaluate the efficiency of the UL for NTM stabilisation, extensive calculations have been performed in the last years, which led amongst others to the choice of the front-steering (FS) launcher [98] instead of the originally envisaged remote-steering (RS) launcher [99] as the reference design for the ITER UL [100]. The FS concept differs from the RS option in that it has movable mirrors facing the plasma, which is a bigger challenge from the engineering point of view. In exchange, the FS launcher allows a better focalisation of the beam in the plasma and hence a higher stabilisation efficiency due to the larger current density achievable. Since the criterion $\eta_{E C C D}>1.2$ can be exceeded by the FS launcher with a huge margin, it becomes possible to extend the possible physics applications of the FS-UL beyond the original goal of NTM stabilisation [101], thus looking for a possible synergy between UL and EL. The investigation of the capabilities of this launcher for extended-physics applications shows [102] that surfaces down to a normalised poloidal radius $\rho_{p} \gtrsim 0.4$ can be reached, producing a higher current density per injected power than by means of the equatorial launcher (see Fig. 4), making the upper launcher attractive for applications like sawtooth control. This study also suggests that a change in the design of the EL to allow counter-CD and to better reach the plasma core could be beneficial to increase the range of physics applications of EC waves for ITER.

Many of the possible sources of error in the theoretical calculations of NTM stabilisation have been considered in the literature, but an overall evaluation of their accuracy is still missing. In addition to the approximation introduced by a linear modelling discussed in 
Sec. II, the design of the UL takes into account the effect of the beam astigmatism [102], multi-beam effects originating from the fact that the beams are launched from different points of the mirror [39] and the influence of steering errors [103]. Deviations with respect to the best achievable stabilisation efficiency predicted by the Rutherford equation can be due to a misalignment of the maximum of the ECCD profile with respect to the resonant surface [104], or can be introduced as effects of the toroidal geometry with respect to slab or cylindrical models [91]. These effects can be investigated also on the basis of cross-machine benchmarking and roughly confirm the criterion $\eta_{E C C D}>1.2$ discussed before. An effect which is usually not taken into account in ray/beam-tracing calculations of the current density driven in the island, but can potentially lead to significantly different values of the current density to be used in the Rutherford equation, is the real island geometry $[105,106]$. An accurate evaluation of these effects under realistic conditions, along the lines of Ref. [107], should be pursued. Also, this kind of calculations could help understand the role of pure heating with respect to current drive [108] under different conditions.

This section is concluded with a glance at the future. The possibility of EC-assisted current ramp-up in DEMO has been studied using a self-consistent coupling of ray tracing, Fokker-Planck and equilibrium solver [109]. The JAERI version of DEMO is a compact reactor where the current induced by the central solenoid needs to be accompanied by externally driven current. Simulations show that reaching a fusion power of $3 \mathrm{GW}$, starting ECCD when the plasma current is at the level of $2 \mathrm{MA}$, is possible and requires a maximum injected EC power of $170 \mathrm{MW}$.

\section{OTHER TOPICS}

The possibility of electron heating due to the absorption of surface flute modes, which are poloidal modes propagating along the surface of the plasma column with a frequency in the EC frequency range $[110,111]$, has recently been discussed in detail $[112,113]$. Absorption of the modes in a toroidal-ripple induced resonance is possible, with possible heating of the plasma edge. The absorption has been shown to be stronger for smaller density gradients. Moreover, the power absorbed near the local resonance is proportional to the square of the ripple amplitude of the external magnetic field.

Technology-related issues are an interesting extension of the theory spectrum beyond the 
more standard subjects discussed in the preceding sections. The dipersion properties of beam-loaded aperiodic corrugated waveguides, which are used in EC applications to prevent the gyrotron interaction from occurring in the latter part of the beam tunnel rather than in the cavity, can be investigated using a method based on an expansion in azimuthal and axial eigenfunctions [114], leading to the corresponding radial dependence for any frequency. This reduces the number of numerical operations involved and allows a calculations of the dispersion relation with few spatial harmonics. The electron dynamics in gyrotron resonators can be described using the canonical perturbation method [115], which allows an elegant solution through a Hamiltonian map, which by construction preserves the invariants of the motion and is numerically more efficient than a standard 4th-order Runge-Kutta solution [116].

More generally, the canonical perturbation method can be used to describe the transient dynamics of electrons interacting with localised waves with continuous spectra, as can be the case in the presence of RF pulses ("aperiodic" perturbation). Approximate invariants of the motion for the non-integrable system can be obtained and used to find an approximate solution of the Vlasov equation for the particle distribution [117].

\section{SUMMARY AND CONCLUSIONS}

"From the pragmatic point of view, the vast majority of phenomena encountered in fusion plasmas are well described by the present approximate theoretical treatments" [3]. This sentence reflects the common view of the fusion community about the present status of the theory of EC waves and has a very sound background. The theoretical research, however, has still to face some challenges. First of all, even within well-established models it is important to ascertain their range of applicability, and the correponding numerical implementation can be made computationally faster or more refined by the inclusion of missing physics. This is particularly true in view of ITER and DEMO, whose development will be accompanied by more and more detailed modelling of the burning plasma. Fast and accurate calculations of different physical ingredients will be essential to this aim. Moreover, several open questions still exists, in a range going from the basic interpretation of the equations for wave propagation in a homogeneous plasma [9] to nonlinear and full-wave modelling of the wave-plasma interaction. 
In this paper, an overview of the current research activity in the field of EC theory has been given. The progress in the basic theory is linked, in particular, to the need of accurate predictions for emission, propagation and absorption of electron Bernstein waves and to the modelling activity for ITER. All the present efforts set the course for a reliable description of EC scenarii in next-generation fusion experiments. 
[1] M. BORNATICI et al., Nucl. Fusion 23, 1153 (1983).

[2] V. ERCKMANN, U. GASPARINO, Plasma Phys. Control. Fusion 36, 1896 (1994).

[3] T. C. LUCE, IEEE Trans. on Plasma Science 30, 734 (2002).

[4] R. PRATER, Phys. Plasmas 11, 2349 (2004).

[5] M. R. O'BRIEN et al., Nucl. Fusion 31, 583 (1991).

[6] R. W. HARVEY et al., Phys. Rev. Lett. 88, 205001 (2002).

[7] K. K. KIROV et al., Plasma Phys. Control. Fusion 44, 2583 (2002).

[8] P. NIKKOLA et al., Nucl. Fusion 43, 1343 (2003).

[9] E. WESTERHOF, Proc. $13^{\text {th }}$ Joint Workshop on ECE and ECRH (Nizhny Novgorod, Russia, May 2004).

[10] L. D. LANDAU, E. M. LIFSHITZ, The Classical Theory of Fields, 4th revised ed., Pergamon Press, 1987.

[11] S. WEINBERG, Phys. Rev. 126, 1899 (1962).

[12] Yu. A. KRATSOV, JETP 28, 769 (1969).

[13] I. B. BERNSTEIN, Phys. Fluids 18, 320 (1975).

[14] U. Bellotti, M. BORNATICI, F. ENGELMANN, Rivista del Nuovo Cimento 20, 1 (1997).

[15] E. MAZZUCATO, Phys. Fluids B 1, 1855 (1989).

[16] S. NOWAK, A. OREFICE, Phys. Fluids B 5, 1945 (1993).

[17] A. G. PEETERS, Phys. Plasmas 3, 4386 (1996).

[18] G. V. PEREVERZEV, Reviews of Plasma Physics 19, B. B. Kadomtsev ed., Consultants Bureau, New York, 1996, p.1.

[19] G. V. PEREVERZEV, Phys. Plasmas 5, 3529 (1998).

[20] E. POLI, G. V. PEREVERZEV, A. G. PEETERS, Phys. Plasmas 6, 5 (1999).

[21] C. TSIRONIS, E. POLI, G. V. PEREVERZEV, Phys. Plasmas 13, 113304 (2006).

[22] See e.g. D. G. SWANSON, Plasma Waves, Academic Press, 1989.

[23] T. M. ANTONSEN, K. R. CHU, Phys. Fluids 25, 1295 (1982).

[24] R. H. COHEN, Phys. Fluids 30, 2442 (1987); Phys. Fluids 31, 421 (1988).

[25] Y. R. LIN-LIU et al., Phys. Plasmas 10, 4064 (2003). 
[26] S. CHOUDHARY, L. B. FELSEN, IEEE Trans. Antennas Propag. AP-21, 827 (1973).

[27] O. MAJ, J. Math. Phys. 46, 083510 (2005).

[28] D. FARINA, Fusion Sci. Technol. 52, 154 (2007).

[29] E. WESTERHOF, L. K. KUZNETSOVA, 30 ${ }^{\text {th }}$ EPS Conf. on Contr. Fusion and Plasma Phys., (St. Petersburg, Russia, July 2003), ECA Vol. 27A, P-3.206.

[30] E. WESTERHOF, L.K. KUZNETSOVA, Plasma Phys. Control. Fusion 49, 1509 (2007).

[31] E. WESTERHOF, Plasma Phys. Control. Fusion 39, 1015 (1997).

[32] A. N. SAVALIEV, A. D. PILIYA, $30^{\text {th }}$ EPS Conf. on Contr. Fusion and Plasma Phys., (St. Petersburg, Russia, July 2003), ECA Vol. 27A, P-2.20.

[33] A. A. BALAKIN et al., Plasma Phys. Rep. 33, 302 (2007).

[34] B. A. TRUBNIKOV, in Plasma Physics and the Problem of Controlled Thermonuclear Reactions, Pergamon Press, 1959, Vol. III, p. 122.

[35] D. FARINA, this issue of Fusion Sci. Technol. (2007).

[36] V. KRIVENSKI, A. OREFICE, J. Plasma Phys. 30, 125 (1983).

[37] I. P. SHKAROFSKY, Phys. Fluids 9, 561 (1966).

[38] R. PRATER et al., Proc. $14^{\text {th }}$ Joint Workshop on ECE and ECRH (Santorini, Greece, May 2006).

[39] E. POLI et al., Proc. $14^{\text {th }}$ Joint Workshop on ECE and ECRH (Santorini, Greece, May 2006).

[40] R. W. HARVEY, M. G. McCOY, Proc. IAEA Technical Committee Meeting on Advances in Simulation and Modeling of Thermonuclear Plasmas, Montreal, Canada, 1992 (IAEA, Vienna, 1993) p. 498.

[41] R. PRATER et al., to be submitted to Nucl. Fusion (2007).

[42] M. ROMÉ et al., Plasma Phys. Control. Fusion 40, 511 (1998).

[43] G. BEKEFI, Radiation Processes in Plasmas, Wiley, 1966.

[44] N. B. MARUSHCHENKO et al., Proc. $14^{\text {th }}$ Joint Workshop on ECE and ECRH (Santorini, Greece, May 2006).

[45] A. P. SMIRNOV et al., Proc. $14^{\text {th }}$ Joint Workshop on ECE and ECRH (Santorini, Greece, May 2006).

[46] N. B. MARUSHCHENKO et al., Fusion Sci. Technol. 50, 395 (2006).

[47] See e.g. F. ALBAJAR et al., Nucl. Fusion 42, 670 (2002). 
[48] F. ALBAJAR et al., Plasma Phys. Control. Fusion 49, 15 (2007).

[49] P. A. ROBINSON, Plasma Phys. Control. Fusion 27, 1037 (1985).

[50] B. A. TRUBNIKOV, Reviews of Plasma Physics 7, M. A. Leontovich ed., Consultants Bureau, New York, 1979, p. 345.

[51] E. POLI, A. G. PEETERS, G. V. PEREVERZEV, Comp. Phys. Comm. 136, 90 (2001).

[52] E. WESTERHOF, A. G. PEETERS, W. L. SCHIPPERS, Rijnhuizen Report RR 92-211 (1992).

[53] L. K. KUZnEtSOVA et al., Proc. $14^{\text {th }}$ Joint Workshop on ECE and ECRH (Santorini, Greece, May 2006).

[54] A. B. KUKUSHKIN et al., Proc. $14^{\text {th }}$ Joint Workshop on ECE and ECRH (Santorini, Greece, May 2006).

[55] R. W. HARVEY, M. G. McCOY, G. D. KERBEL, Phys. Rev. Lett. 62, 426 (1989).

[56] R. KAMENDJE et al., Phys. Plasmas 10, 75 (2003).

[57] R. KAMENDJE et al., Phys. Plasmas 12, 012502 (2005).

[58] D. FARINA et al., Phys. Fluids B 3, 1570 (1991).

[59] A. Yu. KRYACHKO et al., Nucl. Fusion 44, 414 (2004).

[60] A. Yu. KRYACHKO, M. D. TOKMAN, Plasma Phys. Rep. 31, 229 (2005).

[61] A. Yu. KRYACHKO, M. D. TOKMAN, E. WESTERHOF, Phys. Plasmas 13, 072106 (2006).

[62] V. VDOVIN, Proc. $14^{\text {th }}$ Joint Workshop on ECE and ECRH (Santorini, Greece, May 2006).

[63] V. VDOVIN, Proc. $4^{\text {th }}$ IAEA Technical Meeting on ECRH Physics and Technology for ITER (Vienna, Austria, June 2007).

[64] E. D. GOSPODChIKOV, A. G. SHALASHOV, E. V. SUVOROV, Plasma Phys. Control. Fusion 48, 896 (2006).

[65] A. G. SHALASHOV, E. D. GOSPODCHIKOV, E. V. SUVOROV, JETP 103, 480 (2006).

[66] E. D. GOSPODCHIKOV, A. G. SHALASHOV, E. V. SUVOROV, this issue of Fusion Sci. Technol. (2007).

[67] I. B. BERNSTEIN, Phys. Rev. 109, 10 (1958).

[68] A. I. AKHIEZER et al., Plasma Electrodynamics Vol. I (Linear Theory), Pergamon Press, 1975 .

[69] J. PREINHAELTER, V. KOPECKY, J. Plasma Phys. 10, 1 (1973).

[70] See e.g. G. TAYLOR, Fusion Sci. Technol. 52, 119 (2007) and references therein. 
[71] C. B. FOREST et al., Phys. Plasmas 7, 1352 (2000).

[72] A. K. RAM, J. DECKER, Y. PEISSON, J. Plasma Phys. 71, 675 (2005).

[73] A. K. RAM, J. DECKER, Y. PEISSON, Proc. $14^{\text {th }}$ Joint Workshop on ECE and ECRH (Santorini, Greece, May 2006).

[74] J. DECKER, A. K. RAM, Y. PEISSON, Proc. $14^{\text {th }}$ Joint Workshop on ECE and ECRH (Santorini, Greece, May 2006).

[75] T. OHKAWA, Report No. GA-A13847, General Atomics (1976).

[76] N. J. FISCH, A. BOOZER, Phys. Rev. Lett. 45, 720 (1980).

[77] F. VOLPE, N. B. MARUSHCHENKO, Proc. $14^{\text {th }}$ Joint Workshop on ECE and ECRH (Santorini, Greece, May 2006).

[78] P. A. ROBINSON, J. Math. Phys. 27, 1206 (1986).

[79] P. A. ROBINSON, J. Math. Phys. 28, 1203 (1987).

[80] F. CASTEJÓN, S. S. PAVLOV, Phys. Plasmas 13, 072105 (2006).

[81] B. D. FRIED, S. D. CONTE, The Plasma Dispersion Function, Academic Press, 1961.

[82] F. CASTEJÓN et al., Proc. $14^{\text {th }}$ Joint Workshop on ECE and ECRH (Santorini, Greece, May 2006).

[83] A. D. PILIYA, V. I. FEDOROV, JETP 33, 210 (1971).

[84] A. V. TIMOFEEV, Phys. Usp. 174, 555 (2004).

[85] T. H. STIX, Waves in Plasmas, Springer, 1992.

[86] ITER EDA Documentation Series No. 19: Technical Basis for the ITER-FEAT Outline Report, G A0 GDRD 2 01-07-13 R 1.0, published by IAEA, Vienna (2000), Update G A0 GDRD 4 04-01-22 R 0.1 (2004).

[87] O. SAUTER, H. ZOHM, 32th EPS Conf. on Contr. Fusion and Plasma Phys., (Tarragona, Spain, June 2005), ECA Vol. 29C, P-2.059.

[88] G. SAIBENE et al., Proc. 21 ${ }^{\text {th }}$ IAEA Fusion Energy Conference (Chengdu, China, October 2006), IT/P2-14.

[89] F. W. PERKINS et al., Bull. Am. Phys. Soc. 45, 278 (2000).

[90] O. SAUTER, Phys. Plasmas 11, 4808 (2004).

[91] H. ZOHM et al., Proc. $14^{\text {th }}$ Joint Workshop on ECE and ECRH (Santorini, Greece, May 2006).

[92] A. H. KRITZ et al., Proc. $3^{\text {rd }}$ Int. Symp. on Heating in Toroidal Plasmas, CEC Brussels, 
Vol. 2, p. 707 (1982).

[93] K. MATSUDA, IEEE Trans. on Plasma Science 17, 6 (1989).

[94] E. WESTERHOF, Implementation of Toray at JET, Rijnhuizen Report RR-89-183 (1989).

[95] R. W. HARVEY (private communication).

[96] M. R. O'BRIEN et al., Proc. IAEA Technical Committee Meeting on Advances in Simulation and Modeling of Thermonuclear Plasmas, Montreal, Canada, 1992 (IAEA, Vienna, 1993) p. 527.

[97] A. V. ZVONKOV et al., Plasma Phys. Rep. 24, 389 (1998).

[98] R. CHAVAN, M. HENDERSON, F. SANCHEZ, Fus. Eng. Des. 74, 437 (2005).

[99] A. G. A. VERHOEVEN et al., Fus. Eng. Des. 74, 431 (2005).

[100] See e.g. H. ZOHM et al., J. Phys. Conf. Series 25, 234 (2005).

[101] M. A. HENDERSON et al., Proc. 21 ${ }^{\text {th }}$ IAEA Fusion Energy Conference (Chengdu, China, October 2006), IT/P2-15.

[102] G. RAMPONI et al., Fusion Sci. Technol. 52, 193 (2007).

[103] F. VOLPE, J. Phys. Conf. Series 25, 283 (2005).

[104] R. J. LaHAYE et al., Nucl. Fusion 46, 451 (2006).

[105] P. R. da SILVA ROSA, G. GIRUZZI, Plasma Phys. Control. Fusion 42, 755 (2000).

[106] P. R. da SILVA ROSA, L. F. ZIEBELL, Proc. $14^{\text {th }}$ Joint Workshop on ECE and ECRH (Santorini, Greece, May 2006).

[107] K. HAMAmatSU et al., Plasma Phys. Control. Fusion 49, 1955 (2007).

[108] E. WESTERHOF et al., Nucl. Fusion 47, 85 (2007).

[109] M. SATO et al., Proc. $14^{\text {th }}$ Joint Workshop on ECE and ECRH (Santorini, Greece, May 2006).

[110] V. A. GIRKA, I. A. GIRKA, I. V. PAVLENKO, Plasma Phys. Rep. 23, 959 (1997).

[111] V. A. GIRKA, I. A. GIRKA, Plasma Phys. Rep. 28, 682 (2002).

[112] V. O. GIRKA, I. O. GIRKA, Plasma Phys. Rep. 32, 750 (2006).

[113] V. O. GIRKA, I. O. GIRKA, Plasma Phys. Rep. 32, 1047 (2006).

[114] G. P. ANASTASIOU, et al., IEEE Trans. Plasma Sci. 32, 1310 (2004).

[115] Y. KOMINIS et al., Phys. Plasmas 12, 113102 (2005).

[116] O. DUMBRAJS et al., IEEE Trans. Plasma Sci. 34, 673 (2006).

[117] Y. KOMINIS, K. IZANIDIS, A. K. RAM, Phys. Rev. Lett. 96, 025002 (2006). 


\section{Figure captions}

Fig. 1. Maximum and minimum resonant parallel momentum (normalised to $p_{t}=(2 m T)^{1 / 2}$ ) versus major radius $R$ for $R_{0}=6.2 \mathrm{~m}, B_{0}=5.3 \mathrm{~T}, \omega / 2 \pi=170 \mathrm{GHz} T=10 \mathrm{keV}$ and $N_{\|}=0.5$. The solid line corresponds to the fully relativistic case, the dotted line to the weakly relativistic approximation (Reprinted from Ref. [35]).

Fig. 2. (a) Cutoff surfaces $P=0$ and $L=N_{\|}^{2}$ in $1 \mathrm{D}$ and $2 \mathrm{D}$ geometry. (b) Cutoff surfaces $P=0$ and $L=N_{\|}^{2}$ in a poloidal cross section of a tokamak. The regions of $O X$ transformation are marked by arrows (Reprinted from Ref. [64]).

Fig. 3. Imaginary part of the wave vector $k_{i}$ as a function of the magnetic field for the benchmarking case considered in Ref. [38]. The four upper curves correspond to fully relativistic calculations, the two lower curves to weakly relativistic calculations (Courtesy of R. Prater).

Fig. 4. Peak current density per unit power as a function of the normalised radius $\rho_{p}$ for the equatorial launcher (EL) and the extended-physics upper launcher (EPL). For the EL, the injection angles of the top and bottom rows have been tilted poloidally to achieve better localisation (Reprinted from Ref. [102]). 


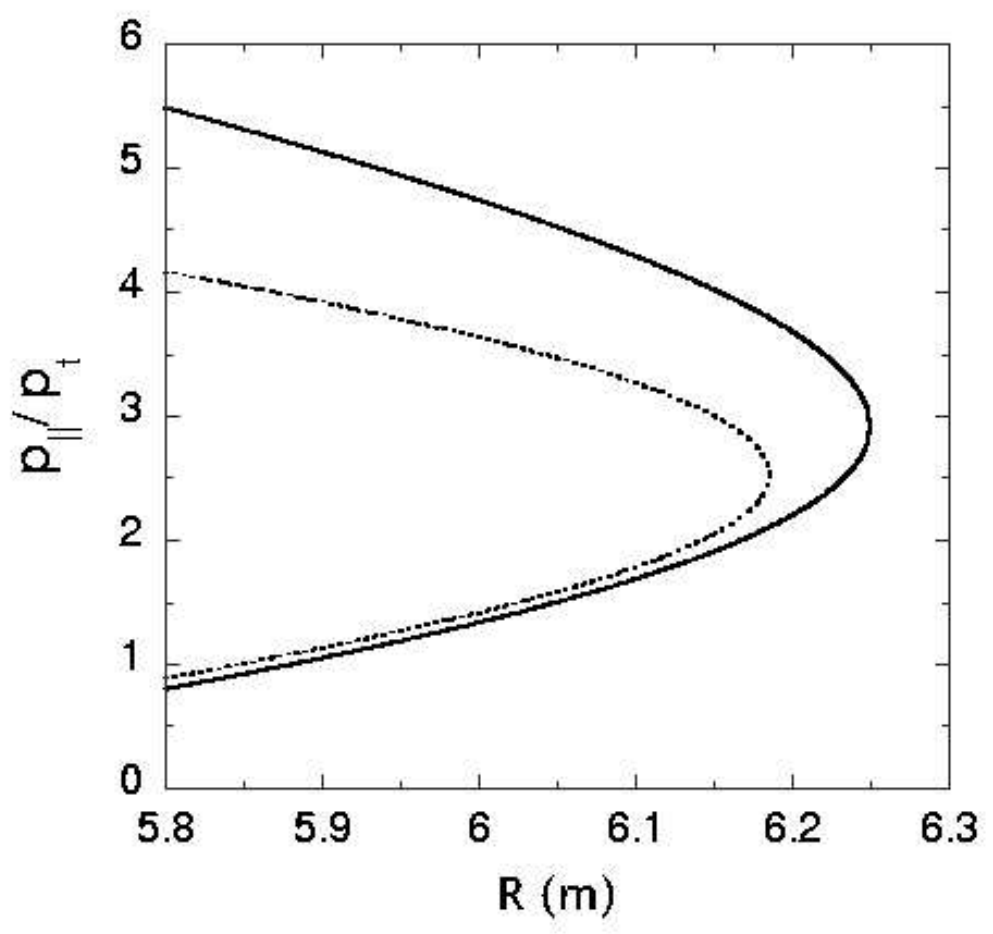



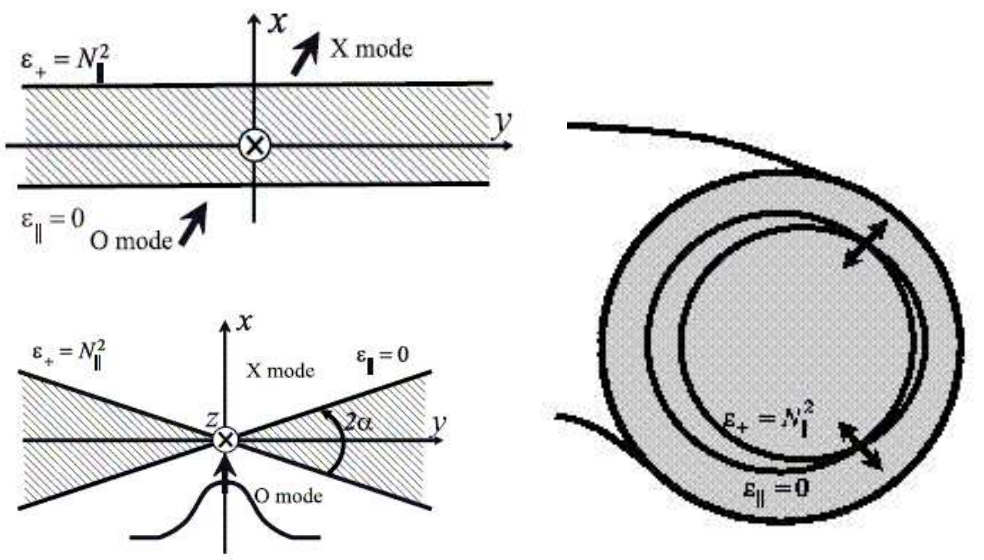


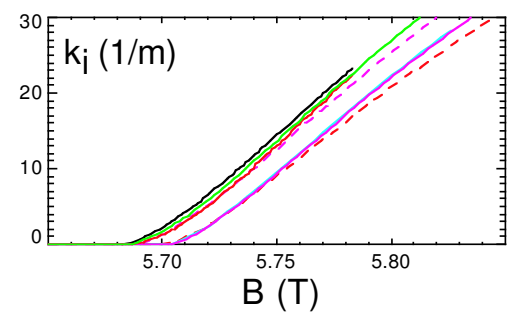




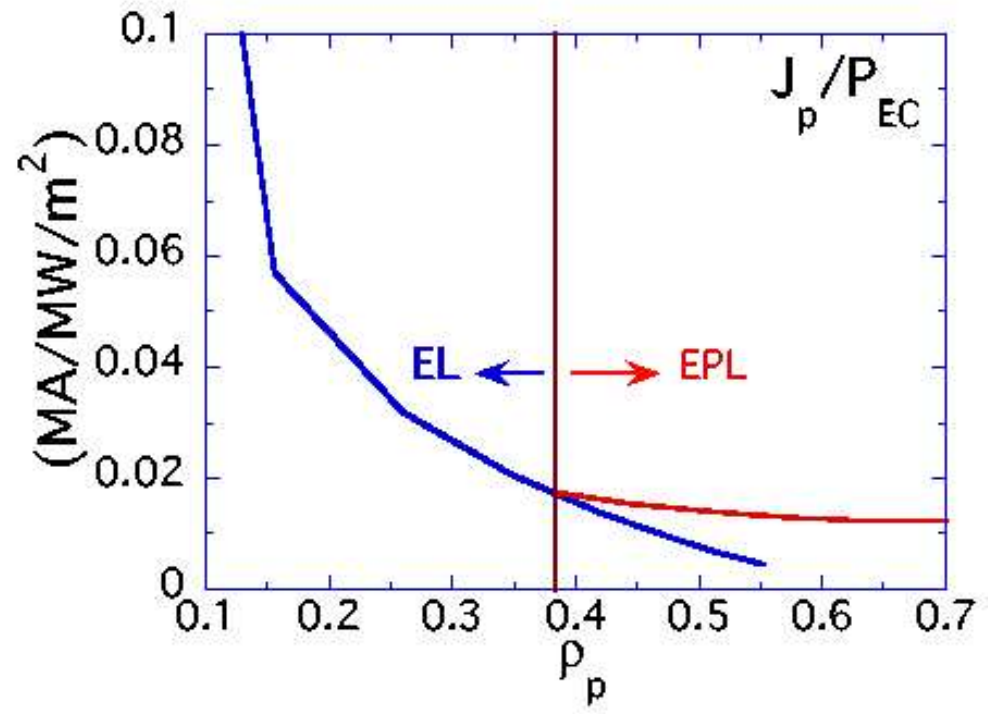

